This is the post print version of the article, which has been published in International journal of number theory. 2018, 14, 1257. http://dx.doi.org/10.1142/S1793042118500781.

D This document has been downloaded from TamPub uta.fi The Institutional Repository of University of Tampere

\title{
Derivation of arithmetical functions under the Dirichlet convolution
}

\author{
Pentti Haukkanen \\ School of Information Sciences, \\ FI-33014 University of Tampere, Finland
}

\begin{abstract}
We present the group-theoretic structure of the classes of multiplicative and firmly multiplicative arithmetical functions of several variables under the Dirichlet convolution, and we give characterizations of these two classes in terms of a derivation of arithmetical functions.
\end{abstract}

Keywords: arithmetical function of several variables, multiplicative function, firmly multiplicative function, derivation, Dirichlet convolution

MSC: $11 \mathrm{~A} 25$

\section{Introduction}

Let $R$ be an integral domain. Let $A_{r}(R):=\left\{f: R^{r} \rightarrow \mathbb{C}\right\}$ denote the set of all arithmetical functions of $r$ variables. An arithmetical function $f \in A_{r}(R)$ is said to be multiplicative if $f(1, \ldots, 1)$ is invertible and

$$
f\left(m_{1} n_{1}, \ldots, m_{r} n_{r}\right)=f\left(m_{1}, \ldots, m_{r}\right) f\left(n_{1}, \ldots, n_{r}\right)
$$


for all positive integers $m_{1}, \ldots, m_{r}$ and $n_{1}, \ldots, n_{r}$ with $\left(m_{1} \cdots m_{r}, n_{1} \cdots n_{r}\right)=$ 1. Clearly, $f(1, \ldots, 1)=1$ if $f$ is multiplicative. Further, a multiplicative function is completely determined by its values at $\left(p^{s_{1}}, \ldots, p^{s_{r}}\right)$, where $p$ runs through all primes and $s_{1}, \ldots, s_{r} \geq 0$. This concept of a multiplicative function coincides with that presented in $[9,12]$ but differs from that used in $[1]$.

We say that an arithmetical function $f \in A_{r}(R)$ is firmly multiplicative if $f(1, \ldots, 1)$ is invertible and

$$
f\left(m_{1} n_{1}, \ldots, m_{r} n_{r}\right)=f\left(m_{1}, \ldots, m_{r}\right) f\left(n_{1}, \ldots, n_{r}\right)
$$

for all positive integers $m_{1}, \ldots, m_{r}$ and $n_{1}, \ldots, n_{r}$ with $\left(m_{1}, n_{1}\right)=\cdots=$ $\left(m_{r}, n_{r}\right)=1$. Clearly, $f(1, \ldots, 1)=1$ if $f$ is firmly multiplicative. Further, a firmly multiplicative function is completely determined by its values at $\left(1, \ldots, 1, p^{s}, 1, \ldots, 1\right)$, where $p$ runs through all primes and $s \geq 1$, i.e., it is completely determined by its values at $\left(n_{1}, \ldots, n_{r}\right)$, where one of $n_{1}, \ldots, n_{r}$ is a prime power $(>1)$ and the others are $=1$. The concept of a firmly multiplicative function coincides with the concept of a multiplicative function presented in [1].

For example, if $f_{1}, \ldots, f_{r}$ are multiplicative functions of one variable, then the arithmetical function $f$ of $r$ variables defined as $f\left(n_{1}, \ldots, n_{r}\right)=$ $f\left(n_{1}\right) \cdots f\left(n_{r}\right)$ is firmly multiplicative. On the other hand, the function $\operatorname{gcd}\left(n_{1}, \ldots, n_{r}\right)$ is multiplicative but not firmly multiplicative for $r \geq 2$. Further examples can be found, e.g., in $[5,10,11]$. A survey on multiplicative arithmetical functions of several variables is presented in [11].

An arithmetical function $f \in A_{r}(R)$ is said to be additive if

$$
f\left(m_{1} n_{1}, \ldots, m_{r} n_{r}\right)=f\left(m_{1}, \ldots, m_{r}\right)+f\left(n_{1}, \ldots, n_{r}\right)
$$

for all positive integers $m_{1}, \ldots, m_{r}$ and $n_{1}, \ldots, n_{r}$ with $\left(m_{1} \cdots m_{r}, n_{1} \cdots n_{r}\right)=$ 1 , and an arithmetical function $f \in A_{r}(R)$ is said to be completely additive if

$$
f\left(m_{1} n_{1}, \ldots, m_{r} n_{r}\right)=f\left(m_{1}, \ldots, m_{r}\right)+f\left(n_{1}, \ldots, n_{r}\right)
$$


for all positive integers $m_{1}, \ldots, m_{r}$ and $n_{1}, \ldots, n_{r}$. We say that an arithmetical function $f \in A_{r}(R)$ is firmly additive if

$$
f\left(m_{1} n_{1}, \ldots, m_{r} n_{r}\right)=f\left(m_{1}, \ldots, m_{r}\right)+f\left(n_{1}, \ldots, n_{r}\right)
$$

for all positive integers $m_{1}, \ldots, m_{r}$ and $n_{1}, \ldots, n_{r}$ with $\left(m_{1}, n_{1}\right)=\cdots=$ $\left(m_{r}, n_{r}\right)=1$. Each completely additive function is firmly additive, and each firmly additive function is additive. Clearly, $f(1, \ldots, 1)=0$ if $f$ is additive.

The Dirichlet convolution of $f, g \in A_{r}(R)$ is defined as

$$
(f * g)\left(n_{1}, \ldots, n_{r}\right)=\sum_{d_{1} \mid n_{1}} \cdots \sum_{d_{r} \mid n_{r}} f\left(d_{1}, \ldots, d_{r}\right) g\left(n_{1} / d_{1}, \ldots, n_{r} / d_{r}\right) .
$$

Let $\delta \in A_{1}(R)$ be defined as $\delta(1)=1$ and $\delta(n)=0$ otherwise, and let $\delta \in A_{r}(R)$ be defined as $\delta\left(n_{1}, \ldots, n_{r}\right)=\delta\left(n_{1}\right) \cdots \delta\left(n_{r}\right)$. Then $\delta$ is the identity under the Dirichlet convolution, and it is firmly multiplicative. The Dirichlet inverse $f^{-1}$ of $f \in A_{r}(R)$ exists if and only if $f(1, \ldots, 1)$ is invertible.

Derivations for arithmetical functions have been presented, e.g., in [1, $2,4,3,6]$. A certain property of multiplicative type functions in terms of derivations is well known $[1,2,4]$, see also $[7,8]$. In this paper we adopt the derivation given in [1] and utilize the method of Rearick [7] to obtain the above mentioned derivation-related property for multiplicative and firmly multiplicative functions, see Theorems 3 and 4 . We obtain a short proof for this property of firmly multiplicative functions given in [1, Theorem 5]. We also show that this property of firmly multiplicative functions actually holds only for firmly multiplicative functions and therefore is, in fact, a characterization of firmly multiplicative functions. An analogous characterization of multiplicative functions is also presented.

We begin by showing the group-theoretic structure of multiplicative and firmly multiplicative functions under the Dirichlet convolution. 


\section{Group-theoretic structure}

Theorem 1. (a) The set of all arithmetical functions $f \in A_{r}(R)$ with $f(1, \ldots, 1)$ invertible is an abelian group under the Dirichlet convolution.

(b) The set of all multiplicative functions forms a subgroup of the abelian group in (a).

(c) The set of all firmly multiplicative functions forms a subgroup of the abelian group in (b).

Proof. We prove only that if $f$ is multiplicative, then $f^{-1}$ is multiplicative. Let $f$ be multiplicative. We proceed by induction on $m_{1} \cdots m_{r} n_{1} \cdots n_{r}$ to prove that

$$
f^{-1}\left(m_{1} n_{1}, \ldots, m_{r} n_{r}\right)=f^{-1}\left(m_{1}, \ldots, m_{r}\right) f^{-1}\left(n_{1}, \ldots, n_{r}\right)
$$

whenever $\left(m_{1} \cdots m_{r}, n_{1} \cdots n_{r}\right)=1$.

Assume that $m_{1} \cdots m_{r} n_{1} \cdots n_{r}=1$. Then $m_{1}=\cdots=m_{r}=n_{1}=\cdots=$ $n_{r}=1$, and since $f$ is multiplicative, $f(1, \ldots, 1)=1$. This implies that $f^{-1}(1, \ldots, 1)=1$, and therefore both sides of (1) equal 1 and thus (1) holds.

Assume that $m_{1} \cdots m_{r} n_{1} \cdots n_{r} \neq 1,\left(m_{1} \cdots m_{r}, n_{1} \cdots n_{r}\right)=1$ and

$$
f^{-1}\left(m_{1}^{\prime} n_{1}^{\prime}, \ldots, m_{r}^{\prime} n_{r}^{\prime}\right)=f^{-1}\left(m_{1}^{\prime}, \ldots, m_{r}^{\prime}\right) f^{-1}\left(n_{1}^{\prime}, \ldots, n_{r}^{\prime}\right)
$$

whenever $\left(m_{1}^{\prime} \cdots m_{r}^{\prime}, n_{1}^{\prime} \cdots n_{r}^{\prime}\right)=1$ and $m_{1}^{\prime} \cdots m_{r}^{\prime} n_{1}^{\prime} \cdots n_{r}^{\prime}<m_{1} \cdots m_{r} n_{1} \cdots n_{r}$. We show that (1) holds. If $m_{1} \cdots m_{r}=1$ or $n_{1} \cdots n_{r}=1$, then (1) holds. Assume that $m_{1} \cdots m_{r} \neq 1$ and $n_{1} \cdots n_{r} \neq 1$. Now, $\left(f * f^{-1}\right)\left(m_{1} n_{1}, \ldots, m_{r} n_{r}\right)=$ 0 , that is,

$f^{-1}\left(m_{1} n_{1}, \ldots, m_{r} n_{r}\right)=-\sum_{\substack{d_{1}\left|m_{1} n_{1}, \ldots, d_{r}\right| m_{r} n_{r} \\ d_{1} \cdots d_{r}>1}} f\left(d_{1}, \ldots, d_{r}\right) f^{-1}\left(m_{1} n_{1} / d_{1}, \ldots, m_{r} n_{r} / d_{r}\right)$ 
Since $\left(m_{1} \cdots m_{r}, n_{1} \cdots n_{r}\right)=1$, the above summation can be written as

$$
-\sum_{\substack{a_{1}\left|m_{1}, \ldots, a_{r}\right| m_{r} \\ b_{1}\left|n_{1} \ldots, \ldots, r_{r}\right| n_{r} \\ a_{1} \cdots a_{r} b_{1} \cdots b_{r}>1}} f\left(a_{1} b_{1}, \ldots, a_{r} b_{r}\right) f^{-1}\left(\left(m_{1} / a_{1}\right)\left(n_{1} / b_{1}\right), \ldots,\left(m_{r} / a_{r}\right)\left(n_{r} / b_{r}\right)\right) .
$$

On the basis of multiplicativity of $f$ and equation (2) this becomes

$$
-\sum_{\substack{a_{1}\left|m_{1}, \ldots, a_{r}\right| m_{r} \\ b_{1}\left|n_{1}, \ldots, b_{r}\right| n_{r} \\ a_{1} \cdots a_{r} b_{1} \cdots b_{r}>1}} f\left(a_{1}, \ldots, a_{r}\right) f\left(b_{1}, \ldots, b_{r}\right) f^{-1}\left(m_{1} / a_{1}, \ldots, m_{r} / a_{r}\right) f^{-1}\left(n_{1} / b_{1}, \ldots, n_{r} / b_{r}\right) .
$$

Arranging the terms we obtain

$$
\begin{aligned}
& f^{-1}\left(m_{1} n_{1}, \ldots, m_{r} n_{r}\right) \\
& =-f^{-1}\left(m_{1}, \ldots, m_{r}\right) \sum_{\substack{b_{1}\left|n_{1}, \ldots, b_{r}\right| n_{r} \\
b_{1} \ldots b_{r}>1}} f\left(b_{1}, \ldots, b_{r}\right) f^{-1}\left(n_{1} / b_{1}, \ldots, n_{r} / b_{r}\right) \\
& -f^{-1}\left(n_{1}, \ldots, n_{r}\right) \sum_{\substack{a_{1}\left|m_{1}, \ldots, a_{r}\right| m_{r} \\
a_{1} \ldots a_{r}>1}} f\left(a_{1}, \ldots, a_{r}\right) f^{-1}\left(m_{1} / a_{1}, \ldots, m_{r} / a_{r}\right) \\
& -\sum_{\substack{a_{1}\left|m_{1}, \ldots, a_{r}\right| m_{r} \\
a_{1} \ldots a_{r}>1}} f\left(a_{1}, \ldots, a_{r}\right) f^{-1}\left(m_{1} / a_{1}, \ldots, m_{r} / a_{r}\right) \\
& \quad \quad \sum_{\substack{b_{1}\left|n_{1}, \ldots, b_{r}\right| n_{r} \\
b_{1} \ldots b_{r}>1}} f\left(b_{1}, \ldots, b_{r}\right) f^{-1}\left(n_{1} / b_{1}, \ldots, n_{r} / b_{r}\right) \\
& \quad-f^{-1}\left(m_{1}, \ldots, m_{r}\right) f^{-1}\left(n_{1}, \ldots, n_{r}\right)+f^{-1}\left(m_{1}, \ldots, m_{r}\right) f^{-1}\left(n_{1}, \ldots, n_{r}\right) \\
& =f^{-1}\left(m_{1}, \ldots, m_{r}\right) f^{-1}\left(n_{1}, \ldots, n_{r}\right) \\
& \left.\quad m_{1}, \ldots, m_{r}\right) f^{-1}\left(n_{1}, \ldots, n_{r}\right) .
\end{aligned}
$$

Thus (1) holds. This shows that $f^{-1}$ is multiplicative.

\section{Derivation}

Let $\psi \in A_{r}(R)$ be a completely additive function. We define the derivation $D_{\psi}: A_{r}(R) \rightarrow A_{r}(R)$ by

$$
D_{\psi}(f)\left(n_{1}, \ldots, n_{r}\right)=f\left(n_{1}, \ldots, n_{r}\right) \psi\left(n_{1}, \ldots, n_{r}\right)
$$


In what follows we write $\mathbf{n}=\left(n_{1}, \ldots, n_{r}\right)$ for the sake of brevity.

We first present the basic properties of the derivation.

Theorem 2. For any $f, g \in A_{r}(R)$ and $c \in R$,

(a) $D_{\psi}(f+g)=D_{\psi}(f)+D_{\psi}(g)$,

(b) $D_{\psi}(f * g)=f * D_{\psi}(g)+g * D_{\psi}(f)$,

(c) $D_{\psi}(c f)=c D_{\psi}(f)$.

Proof. See [1].

We next present the promised characterizations of multiplicative and firmly multiplicative functions in terms of the derivation.

Theorem 3. Let $f \in A_{r}(R)$ be an arithmetical function with $f(1, \ldots, 1)=1$. If $f$ is multiplicative, then $\left(D_{\psi}(f) * f^{-1}\right)(\mathbf{n})=0$ whenever $\mathbf{n}$ is not of the form $\left(p^{s_{1}}, \ldots, p^{s_{r}}\right)$, where $p$ is a prime number and $s_{1}, \ldots, s_{r} \geq 0$. The converse holds provided that $\psi(\mathbf{n}) \neq 0$ for all $\mathbf{n} \neq(1, \ldots, 1)$.

Proof. Assume that $f$ is multiplicative. Assume also that $\mathbf{n}$ is not of the form $\left(p^{s_{1}}, \ldots, p^{s_{r}}\right)$, where $p$ is a prime number and $s_{1}, \ldots, s_{r} \geq 0$. Then $\mathbf{n}$ can be written as $\mathbf{n}=\left(k_{1} m_{1}, \ldots, k_{r} m_{r}\right)$, where $\left(k_{1} \cdots k_{r}, m_{1} \cdots m_{r}\right)=1$ with $k_{1} \cdots k_{r}>1$ and $m_{1} \cdots m_{r}>1$. We show that $\left(D_{\psi}(f) * f^{-1}\right)(\mathbf{n})=0$. Since $f$ is multiplicative, we have

$$
\begin{aligned}
& \left(D_{\psi}(f) * f^{-1}\right)(\mathbf{n}) \\
= & \sum_{d_{1} \mid k_{1} m_{1}} \cdots \sum_{d_{r} \mid k_{r} m_{r}} f\left(d_{1}, \ldots, d_{r}\right) f^{-1}\left(k_{1} m_{1} / d_{1}, \ldots, k_{r} m_{r} / d_{r}\right) \psi\left(d_{1}, \ldots, d_{r}\right) \\
= & \sum_{\substack{a_{1}\left|k_{1} \\
b_{1}\right| m_{1}}} \cdots \sum_{\substack{a_{r}\left|k_{r} \\
b_{r}\right| m_{r}}} f\left(a_{1}, \ldots, a_{r}\right) f\left(b_{1}, \ldots, b_{r}\right) f^{-1}\left(k_{1} / a_{1}, \ldots, k_{r} / a_{r}\right) \\
& \times f^{-1}\left(m_{1} / b_{1}, \ldots, m_{r} / b_{r}\right)\left(\psi\left(a_{1}, \ldots, a_{r}\right)+\psi\left(b_{1}, \ldots, b_{r}\right)\right) .
\end{aligned}
$$


Rearranging the terms we obtain

$$
\begin{aligned}
& \left(D_{\psi}(f) * f^{-1}\right)(\mathbf{n}) \\
& =\sum_{a_{1} \mid k_{1}} \ldots \sum_{a_{r} \mid k_{r}} f\left(a_{1}, \ldots, a_{r}\right) f^{-1}\left(k_{1} / a_{1}, \ldots, k_{r} / a_{r}\right) \psi\left(a_{1}, \ldots, a_{r}\right) \\
& \quad \times \sum_{b_{1} \mid m_{1}} \cdots \sum_{b_{r} \mid m_{r}} f\left(b_{1}, \ldots, b_{r}\right) f^{-1}\left(m_{1} / b_{1}, \ldots, m_{r} / b_{r}\right) \\
& \quad+\sum_{b_{1} \mid m_{1}} \cdots \sum_{b_{r} \mid m_{r}} f\left(b_{1}, \ldots, b_{r}\right) f^{-1}\left(m_{1} / b_{1}, \ldots, m_{r} / b_{r}\right) \psi\left(b_{1}, \ldots, b_{r}\right) \\
& \quad \times \sum_{a_{1} \mid k_{1}} \cdots \sum_{a_{r} \mid k_{r}} f\left(a_{1}, \ldots, a_{r}\right) f^{-1}\left(k_{1} / a_{1}, \ldots, k_{r} / a_{r}\right) \\
& =\left(D_{\psi}(f) * f^{-1}\right)(\mathbf{k}) \delta(\mathbf{m})+\left(D_{\psi}(f) * f^{-1}\right)(\mathbf{m}) \delta(\mathbf{k}) .
\end{aligned}
$$

Since $\mathbf{k} \neq(1, \ldots, 1)$ and $\mathbf{m} \neq(1, \ldots, 1)$, we have $\delta(\mathbf{k})=\delta(\mathbf{m})=0$, and therefore $\left(D_{\psi}(f) * f^{-1}\right)(\mathbf{n})=0$.

Conversely, assume that $\left(D_{\psi}(f) * f^{-1}\right)(\mathbf{n})=0$ whenever $\mathbf{n}$ is not of the form $\left(p^{s_{1}}, \ldots, p^{s_{r}}\right)$, where $p$ is a prime number and $s_{1}, \ldots, s_{r} \geq 0$. We show that $f$ is multiplicative. Let $g \in A_{r}(R)$ be the arithmetical function defined as $g(1, \ldots, 1)=1$ and

$$
g(\mathbf{n})=\prod_{p} f\left(p^{n_{1}(p)}, \ldots, p^{n_{r}(p)}\right)
$$

where $n_{i}=\prod_{p} p^{n_{i}(p)}$ is the canonical factorization of $n_{i}$ for $i=1, \ldots, r$. Then $g$ is multiplicative. We show that $f=g$.

We first show that $D_{\psi}(f) * f^{-1}=D_{\psi}(g) * g^{-1}$. Clearly, $f(\mathbf{n})=g(\mathbf{n})$ if $\mathbf{n}$ is of the form $\left(p^{s_{1}}, \ldots, p^{s_{r}}\right)$, where $p$ is a prime number and $s_{1}, \ldots, s_{r} \geq 0$. This implies that $D_{\psi}(f)(\mathbf{n})=D_{\psi}(g)(\mathbf{n})$ and $f^{-1}(\mathbf{n})=g^{-1}(\mathbf{n})$ if $\mathbf{n}$ is of the form $\left(p^{s_{1}}, \ldots, p^{s_{r}}\right)$, where $p$ is a prime number and $s_{1}, \ldots, s_{r} \geq 0$. Thus $\left(D_{\psi}(f) * f^{-1}\right)(\mathbf{n})=\left(D_{\psi}(g) * g^{-1}\right)(\mathbf{n})$ if $\mathbf{n}$ is of the form $\left(p^{s_{1}}, \ldots, p^{s_{r}}\right)$, where $p$ is a prime number and $s_{1}, \ldots, s_{r} \geq 0$. Since $g$ is multiplicative, on the basis of the first part of this theorem $\left(D_{\psi}(g) * g^{-1}\right)(\mathbf{n})=0$ if $\mathbf{n}$ is not of the form $\left(p^{s_{1}}, \ldots, p^{s_{r}}\right)$, where $p$ is a prime number and $s_{1}, \ldots, s_{r} \geq 0$. Thus, by the assumption on $f,\left(D_{\psi}(f) * f^{-1}\right)(\mathbf{n})=\left(D_{\psi}(g) * g^{-1}\right)(\mathbf{n})$ for all $\mathbf{n}$. 
We now show that $f(\mathbf{n})=g(\mathbf{n})$ for all $\mathbf{n}=\left(n_{1}, \ldots, n_{r}\right)$ applying induction on $\Omega\left(n_{1}\right)+\cdots+\Omega\left(n_{r}\right)$, where $\Omega\left(n_{i}\right)$ is the total number of prime divisors of $n_{i}$ each counted according to its multiplicity with $\Omega(1)=0$. If $\Omega\left(n_{1}\right)+$ $\cdots+\Omega\left(n_{r}\right)=0$, then $\mathbf{n}=(1, \ldots, 1)$ and $f(\mathbf{n})=g(\mathbf{n})=1$. Assume that $f(\mathbf{n})=g(\mathbf{n})$ for $\Omega\left(n_{1}\right)+\cdots+\Omega\left(n_{r}\right)<k$. Then $f^{-1}(\mathbf{n})=g^{-1}(\mathbf{n})$ for $\Omega\left(n_{1}\right)+$ $\cdots+\Omega\left(n_{r}\right)<k$. Let $\mathbf{m}$ be such that $\Omega\left(m_{1}\right)+\cdots+\Omega\left(m_{r}\right)=k$. We show that $f(\mathbf{m})=g(\mathbf{m})$. We have shown that $\left(D_{\psi}(f) * f^{-1}\right)(\mathbf{m})=\left(D_{\psi}(g) * g^{-1}\right)(\mathbf{m})$, which means that

$$
\begin{aligned}
& \sum_{d_{1}\left|m_{1}, \ldots, d_{r}\right| m_{r}} f\left(d_{1}, \ldots, d_{r}\right) f^{-1}\left(m_{1} / d_{1}, \ldots, m_{r} / d_{r}\right) \psi\left(d_{1}, \ldots, d_{r}\right) \\
= & \sum_{d_{1}\left|m_{1}, \ldots, d_{r}\right| m_{r}} g\left(d_{1}, \ldots, d_{r}\right) g^{-1}\left(m_{1} / d_{1}, \ldots, m_{r} / d_{r}\right) \psi\left(d_{1}, \ldots, d_{r}\right) .
\end{aligned}
$$

Since $\psi(1, \ldots, 1)=0$, we have

$$
\begin{aligned}
& f(\mathbf{m}) \psi(\mathbf{m})+\sum_{\begin{array}{c}
d_{1}\left|m_{1}, \ldots, d_{r}\right| m_{r} \\
\left(d_{1}, \ldots, d_{r}\right) \neq(1, \ldots, 1) \\
\left(d_{1}, \ldots, d_{r}\right) \neq\left(m_{1}, \ldots, m_{r}\right)
\end{array}} f\left(d_{1}, \ldots, d_{r}\right) f^{-1}\left(m_{1} / d_{1}, \ldots, m_{r} / d_{r}\right) \psi\left(d_{1}, \ldots, d_{r}\right) \\
& =g(\mathbf{m}) \psi(\mathbf{m})+\sum_{\begin{array}{c}
d_{1}\left|m_{1}, \ldots, d_{r}\right| m_{r} \\
\left(d_{1}, \ldots, d_{r}\right) \neq(1, \ldots, 1) \\
\left(d_{1}, \ldots, d_{r}\right) \neq\left(m_{1}, \ldots, m_{r}\right)
\end{array}} g\left(d_{1}, \ldots, d_{r}\right) g^{-1}\left(m_{1} / d_{1}, \ldots, m_{r} / d_{r}\right) \psi\left(d_{1}, \ldots, d_{r}\right) .
\end{aligned}
$$

Since $f(\mathbf{n})=g(\mathbf{n})$ and $f^{-1}(\mathbf{n})=g^{-1}(\mathbf{n})$ for $\Omega\left(n_{1}\right)+\cdots+\Omega\left(n_{r}\right)<k$, the summations above are equal. Thus $f(\mathbf{m}) \psi(\mathbf{m})=g(\mathbf{m}) \psi(\mathbf{m})$, and since $\psi(\mathbf{m}) \neq 0$, we have $f(\mathbf{m})=g(\mathbf{m})$. We have thus shown by induction that $f=g$. Since $g$ is multiplicative, $f$ is multiplicative. This completes the proof.

Theorem 4. Let $f \in A_{r}(R)$ be an arithmetical function with $f(1, \ldots, 1)=$ 1. If $f$ is firmly multiplicative, then $\left(D_{\psi}(f) * f^{-1}\right)(\mathbf{n})=0$ whenever $\mathbf{n}=$ $\left(n_{1}, \ldots, n_{r}\right)$ is not of the form $\mathbf{n}=\left(1, \ldots, 1, p^{s}, 1, \ldots, 1\right)$, where $p$ is a prime number and $s \geq 0$ (i.e. $\left(D_{\psi}(f) * f^{-1}\right)(\mathbf{n})=0$ unless one of $n_{1}, \ldots, n_{r}$ is a prime power $(\geq 1)$ and the others are $=1)$. The converse holds provided that $\psi(\mathbf{n}) \neq 0$ for all $\mathbf{n} \neq(1, \ldots, 1)$. 
Proof. Assume that $f$ is firmly multiplicative. Assume also that $\mathbf{n}$ is not of the form $\left(1, \ldots, 1, p^{s}, 1, \ldots, 1\right)$, where $p$ is a prime number and $s \geq 0$. Then $\mathbf{n}$ can be written as $\mathbf{n}=\left(k_{1} m_{1}, \ldots, k_{r} m_{r}\right)$, where $\left(k_{1}, m_{1}\right)=\cdots=\left(k_{r}, m_{r}\right)=1$ with $k_{1} \cdots k_{r}>1$ and $m_{1} \cdots m_{r}>1$. We show that $\left(D_{\psi}(f) * f^{-1}\right)(\mathbf{n})=0$. Since $f$ is firmly multiplicative, we have

$$
\begin{aligned}
& \left(D_{\psi}(f) * f^{-1}\right)(\mathbf{n}) \\
= & \sum_{d_{1} \mid k_{1} m_{1}} \cdots \sum_{d_{r} \mid k_{r} m_{r}} f\left(d_{1}, \ldots, d_{r}\right) f^{-1}\left(k_{1} m_{1} / d_{1}, \ldots, k_{r} m_{r} / d_{r}\right) \psi\left(d_{1}, \ldots, d_{r}\right) \\
= & \sum_{\substack{a_{1}\left|k_{1} \\
b_{1}\right| m_{1}}} \cdots \sum_{\substack{a_{r}\left|k_{r} \\
b_{r}\right| m_{r}}} f\left(a_{1}, \ldots, a_{r}\right) f\left(b_{1}, \ldots, b_{r}\right) f^{-1}\left(k_{1} / a_{1}, \ldots, k_{r} / a_{r}\right) \\
& \times f^{-1}\left(m_{1} / b_{1}, \ldots, m_{r} / b_{r}\right)\left(\psi\left(a_{1}, \ldots, a_{r}\right)+\psi\left(b_{1}, \ldots, b_{r}\right)\right) \\
= & \left(D_{\psi}(f) * f^{-1}\right)(\mathbf{k}) \delta(\mathbf{m})+\left(D_{\psi}(f) * f^{-1}\right)(\mathbf{m}) \delta(\mathbf{k}) .
\end{aligned}
$$

Since $\mathbf{k} \neq(1, \ldots, 1)$ and $\mathbf{m} \neq(1, \ldots, 1)$, we have $\delta(\mathbf{k})=\delta(\mathbf{m})=0$, and therefore $\left(D_{\psi}(f) * f^{-1}\right)(\mathbf{n})=0$.

Conversely, assume that $\left(D_{\psi}(f) * f^{-1}\right)(\mathbf{n})=0$ whenever $\mathbf{n}$ is not of the form $\left(1, \ldots, 1, p^{s}, 1, \ldots, 1\right)$, where $p$ is a prime number and $s \geq 0$. We show that $f$ is firmly multiplicative. Let $g \in A_{r}(R)$ be the arithmetical function defined as $g(1, \ldots, 1)=1$ and

$$
g(\mathbf{n})=\prod_{p}\left(f\left(p^{n_{1}(p)}, 1, \ldots, 1\right) f\left(1, p^{n_{2}(p)}, 1, \ldots, 1\right) \cdots f\left(1, \ldots, 1, p^{n_{r}(p)}\right)\right)
$$

where $n_{i}=\prod_{p} p^{n_{i}(p)}$ is the canonical factorization of $n_{i}$ for $i=1,2, \ldots, r$. Then $g$ is firmly multiplicative. We show that $f=g$.

We first show that $D_{\psi}(f) * f^{-1}=D_{\psi}(g) * g^{-1}$. Clearly, $f(\mathbf{n})=g(\mathbf{n})$ if $\mathbf{n}$ is of the form $\left(1, \ldots, 1, p^{s}, 1, \ldots, 1\right)$, where $p$ is a prime number and $s \geq 0$. This implies that $D_{\psi}(f)(\mathbf{n})=D_{\psi}(g)(\mathbf{n})$ and $f^{-1}(\mathbf{n})=g^{-1}(\mathbf{n})$ if $\mathbf{n}$ is of the form $\left(1, \ldots, 1, p^{s}, 1, \ldots, 1\right)$, where $p$ is a prime number and $s \geq 0$. Thus $\left(D_{\psi}(f) * f^{-1}\right)(\mathbf{n})=\left(D_{\psi}(g) * g^{-1}\right)(\mathbf{n})$ if $\mathbf{n}$ is of the form $\left(1, \ldots, 1, p^{s}, 1, \ldots, 1\right)$, where $p$ is a prime number and $s \geq 0$. Since $g$ is firmly multiplicative, on the basis of the first part of this theorem $\left(D_{\psi}(g) * g^{-1}\right)(\mathbf{n})=0$ if $\mathbf{n}$ is not of the 
form $\left(1, \ldots, 1, p^{s}, 1, \ldots, 1\right)$, where $p$ is a prime number and $s \geq 0$. Thus, by the assumption on $f,\left(D_{\psi}(f) * f^{-1}\right)(\mathbf{n})=\left(D_{\psi}(g) * g^{-1}\right)(\mathbf{n})$ for all $\mathbf{n}$.

We can show that $f(\mathbf{n})=g(\mathbf{n})$ for all $\mathbf{n}=\left(n_{1}, \ldots, n_{r}\right)$ applying induction on $\Omega\left(n_{1}\right)+\cdots+\Omega\left(n_{r}\right)$ exactly in the same way as in the proof of Theorem 3 . Thus $f=g$, and since $g$ is firmly multiplicative, $f$ is firmly multiplicative. This completes the proof.

Acknowledgment The author wishes thank the referee for carefully reading the paper and useful comments.

\section{References}

[1] E. Alkan, A. Zaharescu and M. Zaki, Arithmetical functions in several variables, Int. J. Number Theory 1(3) (2005) 383-399.

[2] E. Alkan, A. Zaharescu and M. Zaki, Unitary convolution for arithmetical functions in several variables, Hiroshima Math. J. 36(1) (2006) $113-124$.

[3] T. Komatsu, V. Laohakosol and P. Ruengsinsub, Independence measures of arithmetic functions, J. Number Theory 131(1) (2011) 1-17.

[4] V. Laohakosol, N. Pabhapote and N. Wechwiriyakul, Arithmetic functions and operators, ScienceAsia 27 (2001) 199-202.

[5] V. A. Liskovets, A multivariate arithmetic function of combinatorial and topological significance, Integers 10 (2010) 155-177.

[6] N. Pabhapote and V. Laohakosol, Distributive property of completely multiplicative functions, Lith. Math. J. 50(3) (2010) 312-322.

[7] D. Rearick, Operators on algebras of arithmetic functions, Duke Math. J. 35 (1968) 761-766. 
[8] D. Rearick, The trigonometry of numbers, Duke Math. J. 35 (1968) $767-776$.

[9] R. Sivaramakrishnan, Classical Theory of Arithmetic Functions, Monographs and Textbooks in Pure and Applied Mathematics, Vol. 126. (Marcel Dekker, Inc., 1989).

[10] L. Tóth, Some remarks on a paper of V. A. Liskovets, Integers 12 (2012) $97-111$.

[11] L. Tóth, Multiplicative arithmetic functions of several variables: A survey, in vol. Mathematics Without Boundaries, Surveys in Pure Mathematics, T. M. Rassias, P. M. Pardalos (eds.), (Springer, 2014), pp. $483-514$.

[12] R. Vaidyanathaswamy, The theory of multiplicative arithmetic functions, Trans. Amer. Math. Soc. 33 (1931) 579-662. 